\title{
An Improved Evaluation of the Neutron Background in the PandaX-II Experiment
}

Qiuhong Wang*1,13, Abdusalam Abdukerim², Wei Chen², Xun Chen ${ }^{2}$, Yunhua Chen ${ }^{3}$, Xiangyi Cui ${ }^{2}$, Yingjie Fan ${ }^{4}$, Deqing Fang ${ }^{1}$, Changbo Fu² ${ }^{2}$ Lisheng Geng ${ }^{5}$, Karl Giboni ${ }^{2}$, Franco Giuliani ${ }^{2}$, Linhui Gu ${ }^{2}$, Xuyuan $\mathrm{Guo}^{3}$, Ke $\mathrm{Han}^{2}$, Changda $\mathrm{He}^{2}$, Di Huang 2 , Yan Huang ${ }^{3}$, Yanlin Huang ${ }^{6}$, Zhou Huang 2 , Peng $\mathrm{Ji}^{4}$, Xiangdong $\mathrm{Ji}^{2,7}$, Yonglin $\mathrm{Ju}^{8}$, Yihui Lai ${ }^{2}$, Kun Liang ${ }^{2}$, Huaxuan Liu ${ }^{8}$, Jianglai Liu ${ }^{\dagger 2,7}$, Wenbo $\mathrm{Ma}^{2}$, Yugang $\mathrm{Ma}^{1}$, Yajun $\mathrm{Mao}^{9}$, Yue Meng ${ }^{2}$, Parinya Namwongsa ${ }^{2}$, Kaixiang $\mathrm{Ni}^{2}$, Jinhua Ning ${ }^{3}$, Xuyang Ning ${ }^{2}$, Xiangxiang Ren ${ }^{8,2}$, Changsong Shang ${ }^{3}$, Lin $\mathrm{Si}^{2}$, Andi Tan ${ }^{10}$, Anqing Wang ${ }^{11}$, Hongwei Wang ${ }^{1}$, Meng Wang ${ }^{11}$, Siguang Wang ${ }^{9}$, Xiuli Wang ${ }^{8}$, Zhou Wang ${ }^{10,12,2}$, Mengmeng $\mathrm{Wu}^{4}$, Shiyong $\mathrm{Wu}^{3}$, Jingkai Xia ${ }^{2}$, Mengjiao Xiao ${ }^{10,12}$, Pengwei Xie ${ }^{7}$, Binbin Yan ${ }^{11}$, Jijun Yang ${ }^{2}$, Yong Yang ${ }^{2}$, Chunxu Yu ${ }^{4}$, Jumin Yuan ${ }^{11}$, Dan Zhang ${ }^{10}$, Hongguang Zhang ${ }^{2}$, Tao Zhang ${ }^{2}$, Li Zhao ${ }^{2}$, Qibin Zheng ${ }^{6}$, Jifang Zhou ${ }^{3}$, Ning Zhou ${ }^{2}$, and Xiaopeng Zhou ${ }^{5}$

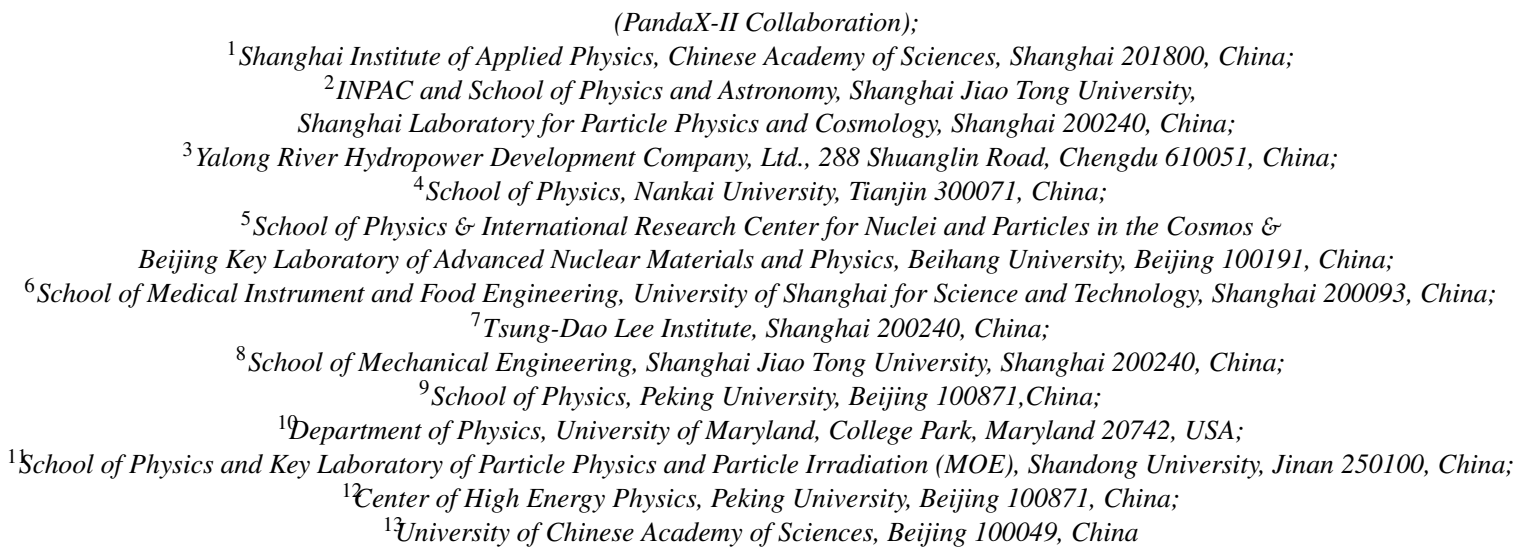

In dark matter direct detection experiments, neutron is a serious source of background, which can mimic the dark matter-nucleus scattering signals. In this paper, we present an improved evaluation of the neutron background in the PandaX-II dark matter experiment by a novel approach. Instead of fully relying on the Monte Carlo simulation, the overall neutron background is determined from the neutron-induced high energy signals in the data. In addition, the probability of producing a dark-matter-like background per neutron is evaluated with a complete Monte Carlo generator, where the correlated emission of neutron(s) and $\gamma(\mathrm{s})$ in the $(\alpha, \mathrm{n})$ reactions and spontaneous fissions is taken into consideration. With this method, the neutron backgrounds in the Run 9 (26-ton-day) and Run 10 (28-ton-day) data sets of PandaX-II are estimated to be $0.66 \pm 0.24$ and $0.47 \pm 0.25$ events, respectively.

dark matter, direct detection, xenon, neutron background, high energy gamma, neutron generator PACS number(s): $95.35 .+\mathrm{d}, 29.40 . \mathrm{n}, 28.20 . \mathrm{v}$

*Corresponding author: wangqiuhong@ sinap.ac.cn

†Spokesperson: jianglai.liu@ @jtu.edu.cn 


\section{Introduction}

Modern astronomical and cosmological observations have firmly established the existence of dark matter (DM) in our Galaxy and the Universe [1]. Since the seminal paper by Goodman and Witten [2], there has been an intensive global effort of DM direct detection, searching for the nuclear recoil (NR) of the atomic nucleus in the target induced by galactic DM particles [3-5].

As one of such experiments, the PandaX experiment, located at the China Jinping Underground Laboratory [6] uses liquid xenon as the DM direct detection target $[7,8]$. The second phase of this experiment, PandaX-II [9], contains a total of 1.1 tons of liquid xenon in a stainless steel (SS) cryostat with low levels of radioactivity [10]. Approximately $580 \mathrm{~kg}$ of liquid xenon is confined inside the $60-\mathrm{cm}$ high and $60-\mathrm{cm}$ diametral dual phase xenon time projection chamber (TPC) with Hamamatsu R11410 photomultipliers (PMTs) located at the top and bottom. For each recoil event, the prompt scintillation photons $(S 1)$ and the delayed electroluminescence photons $(S 2)$ from ionized electrons can be detected by those two arrays of PMTs. Highly reflective polytetrafluoroethylene (PTFE) layers are installed surrounding the TPC to enhance the photon collection efficiency. So far, PandaX-II has released two data sets, the Run 9 (79.6 live days, 26-ton-day exposure) [9] and Run 10 (77.1 live days, 28-ton-day exposure) [11].

From the background point of view, the charge ratio of $S 2$ to $S 1$ offers a strong discriminant for the NR signals against the electron recoil (ER) background events from $\gamma \mathrm{s}$ and $\beta \mathrm{s}$. On the other hand, the neutrons could produce single-site scattering NR (SSNR) events that highly resemble the DM interactions. External neutrons can be reduced to a negligible level via carefully designed shielding. However, neutrons can be produced internally from radioactivities in the detector materials through two main reactions, the $(\alpha, \mathrm{n})$ reaction and spontaneous fission (SF) of ${ }^{238} \mathrm{U}$, and bring about a nonnegligible background to the DM detection.

Traditionally, neutron background evaluation is heavily simulation-based. In this paper, we present a novel data-driven method to evaluate the neutron background in PandaX-II, utilizing neutron-induced high energy gamma (HEG) events as the normalization. The rest of this paper is organized as follows. In sect. 2, we explain our improved method of the SSNR background evaluation. In sect. 3, we use the ${ }^{241} \mathrm{Am}-{ }^{-} \mathrm{Be}(\mathrm{AmBe})$ neutron calibration data to understand the SSNR and HEG events. In sect. 4, we discuss our improved neutron generator model and establish an expected ratio between the two types of events originating from the detector materials. A new estimation of the neutron background level in PandaX-II is given in sect. 5, followed by a summary in sect. 6.

\section{Method}

Traditionally, the SSNR background estimation for DM experiments has the following three ingredients, 1) radioactivities of detector materials, 2) a model which converts the radioactivities to the number of neutrons produced and their energy spectrum, and 3) a detector Monte Carlo (MC) simulation which uses the model as the event generator and carries out detailed simulation of neutron interactions and the detector response. The SSNR background is calculated as

$$
\begin{aligned}
N_{\mathrm{ssnr}} & =\sum_{i j} P_{\mathrm{ssnr}, i j} \times n_{i j} \times T \\
n_{i j} & =Y_{i j} \times A_{i j} \times M_{i} .
\end{aligned}
$$

In these expressions, $n_{i j}$ is the neutron production rate from a detector component $i$ due to a given radionuclide or a reaction chain $j$, calculated by combining the radioactivity per unit mass $A_{i j}$, the neutron yield $Y_{i j}$, and the component mass $M_{i}$. $Y_{i j}$ is typically computed by the SOURCES-4A code [12], which calculates the $(\alpha, \mathrm{n})$ and SF neutron production rates and spectra using evaluated libraries. $P_{\mathrm{ssnr}, i j}$ is the probability of producing an SSNR signal per neutron calculated by the detector $\mathrm{MC}$ and $T$ is the duration of the measurement.

The above method has two serious drawbacks. First, SOURCES-4A and the resulting neutron generator treat every neutron as an isolated particle. This is, in fact, a biased assumption - there is a high probability that the neutron(s) is generated in association with $\gamma(\mathrm{s})$ in the $(\alpha, \mathrm{n})$ and SF reactions, leading to multi-site scatterings and mixed ER-NR energy depositions. Second, the uncertainty in radioassay of detector materials $A_{i j}$ would be directly translated into a normalization uncertainty in the SSNR rate, which lacks experimental handle.

In light of the above, we developed a new method to estimate the neutron background. A complete neutron generator was developed which incorporates the correlated emission of neutron(s) and $\gamma(\mathrm{s})$. This allows us to establish a more robust relationship between the SSNR events and the neutroninduced HEG events. Then the SSNR background can be estimated as

$$
N_{\mathrm{ssnr}}=N_{\mathrm{heg}} \times R_{\mathrm{mc}}=N_{\mathrm{heg}} \times\left(\frac{\sum_{i j} P_{\mathrm{ssnr}, i j} \times n_{i j}}{\sum_{i j} P_{\text {heg }, i j} \times n_{i j}}\right)
$$

where $N_{\text {heg }}$ is the number of identified HEG events from DM data, which serves as an in situ normalization. $R_{\mathrm{mc}}$ is an MC-based ratio between the summed SSNR and HEG events from individual components, obtained by folding the neutron 
production rate $n_{i j}$ and the probability to produce an SSNR (HEG) event, $P_{\mathrm{ssnr}, i j}\left(P_{\mathrm{heg}, i j}\right)$. In what follows, we shall first use the AmBe calibration to understand the connection between SSNR and HEG events, then discuss our evaluation of $R_{\mathrm{mc}}$ and $N_{\text {heg }}$ in turn.

\section{The AmBe calibration}

\subsection{The AmBe neutron source}

Two AmBe sources with the identical design were fabricated by the Atomic High Technology Co., Ltd. ${ }^{1)}$. The sources produce neutrons via the $(\alpha, \mathrm{n})$ reaction

$$
{ }^{9} \mathrm{Be}+\alpha \rightarrow \mathrm{n}+{ }^{12} \mathrm{C}^{*} .
$$

Two channels, $\left(\alpha, \mathrm{n}_{0}\right)$ and $\left(\alpha, \mathrm{n}_{1}\right)$, dominate the reaction, in which ${ }^{12} \mathrm{C}^{*}$ is produced in the ground and the first excited state, respectively. The latter channel emits a single deexcitation $\gamma$ ray of $4.4 \mathrm{MeV}$ together with the neutron. The neutron energy spectra of the two channels can be calculated by combining a stand-alone Geant4-based $[13,14]$ simulation (energy loss process of $\alpha$ ) and the JENDL database [15] (neutron production) and are shown in Figure 1. One of the two AmBe sources was deployed into the Daya Bay detector [16], where the simulated energy spectra were validated through measurement [17]. The other one was used for the calibration of PandaX-II.

The AmBe source was deployed inside two separate horizontal PTFE tubes encircling the cryostat, at a vertical height of $1 / 4$ (lower) and 3/4 (upper) of the TPC. The AmBe calibration runs in PandaX-II were performed more than 20 times, interspersed among the DM data taking, with the source located at different locations in the loops. In total,

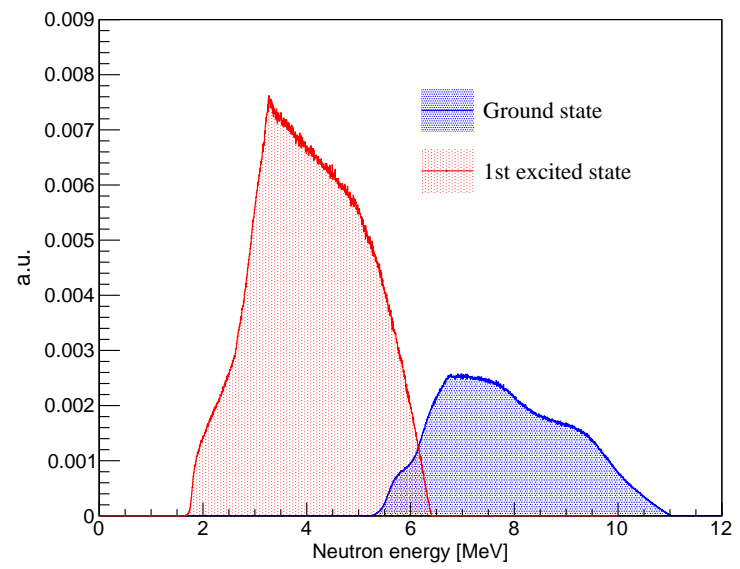

Figure 1 The neutron energy spectrum of the AmBe source from simulation, with the two histograms corresponding to $\left(\alpha, \mathrm{n}_{0}\right)$ (red) and $\left(\alpha, \mathrm{n}_{1}\right)$ (blue).

1) For details, see http://en.atom-hitech.com/product/51.html.
6.8 and 22.8 days of AmBe data were taken in Run 9 and Run 10, respectively, with a source-induced rate of approximately $2 \mathrm{~Hz}$. For different source locations, no significant difference was identified in the data or the MC, so we made no distinction in the calibration data analysis.

\subsection{AmBe data}

AmBe neutrons can produce the SSNR signal through elastic neutron-xenon-nucleus scattering if the scattering happens to be single-site in the TPC. More often, however, AmBe neutrons would scatter with xenon nuclei inelastically, producing NR signals mixed with ER signals from the nuclear de-excitation. In both cases above, the energy deposition is "prompt", and may be further mixed with the "prompt" ER signals produced by the $4.4 \mathrm{MeV} \gamma$ ray. On the other hand, after being thermalized, neutrons are also likely to be captured by xenon nuclei, which produces "delayed" high energy $\gamma$ rays with a few $\mu$ s delay time. These neutron-induced HEGs, regardless if produced promptly or by delayed capture, are related to the SSNR events through the parent neutrons.

In the AmBe calibration data sets, SSNR events are selected using identical cuts for dark matte data selection [9, 11], with $S 1$ from 3 to 45 photoelectrons (PE) and $S 2$ from $100 \mathrm{PE}$ (raw) to $10000 \mathrm{PE}$ (corrected for position uniformity). These cuts correspond to an electron-equivalent energy range between 1 and $10 \mathrm{keV}_{e e}$ approximately. The same fiducial volume (FV) cuts in Run 9 and Run 10 are adopted here.

The HEGs are easy to be identified because their total energy can be significantly higher than those from natural radioactivities. In addition, $\gamma \mathrm{s}$ can be produced at different times (prompt or delayed) and tend to have multiple scatterings with xenon, resulting in multiple $S 1$ s and $S 2$ s. A small fraction of them contain a separated NR signal before the HEGs from the delayed capture. An example of such waveforms is shown in Figure 2.

In the HEG event selection, the data quality cuts developed

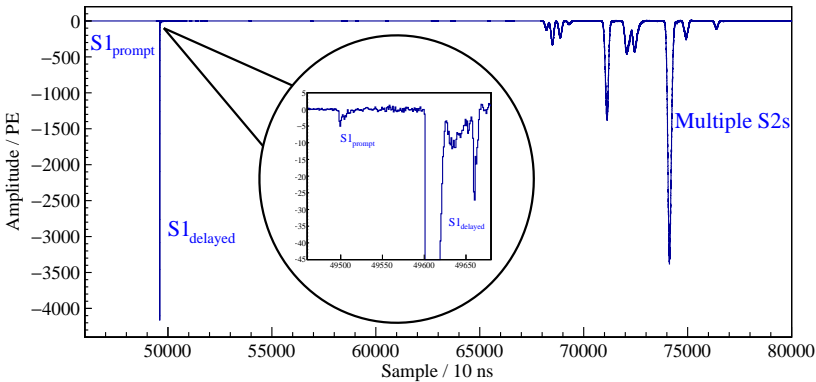

Figure 2 An example of waveforms of high energy neutron capture events in AmBe runs, in which the prompt NR occurred before the delayed HEGs. 
in Ref. [18] are inherited for each $S 1$ and $S 2$. For each scattering, the horizontal position is reconstructed using the socalled PAF reconstruction [9]. The approximate vertical positions of the scatterings are estimated by pairing these $S 2 \mathrm{~s}$ with the maximum $S 1^{2}$ ). Since we are interested in the HEGs with energies larger than $5.0 \mathrm{MeV}\left(\mathrm{Q}\right.$ value of ${ }^{208} \mathrm{Tl}$, the maximum ER energy by natural radioactivity), there is no need of a restrictive FV cut for background rejection. Also to avoid the bias in the horizontal position reconstruction caused by the PMT saturation for HEGs, we select all $S$ 2s within an extended FV (EFV) with a loose radius-square cut of $r^{2}<1000 \mathrm{~cm}^{2}$ and a vertical position cut of 1.7 and $3.3 \mathrm{~cm}$ away from the cathode and gate, respectively, resulting in a target xenon mass of $502 \mathrm{~kg}$. The total energy is computed by combining all $S 1$ s and $S 2$ s within the EFV ${ }^{3)}$. To avoid saturation of the PMTs at this high energy, the $S 2$ signals in the bottom PMT array $\left(S 2_{\mathrm{b}}\right)$, scaled by a factor of 3.5 , are used in the reconstruction. A corrected energy, $E_{\text {corr }}$, is obtained by further applying a polynomial correction to align the energy of the identified $\gamma$ events, i.e. $609 \mathrm{keV}\left({ }^{214} \mathrm{Bi}\right), 911 / 934 \mathrm{keV}$ $\left({ }^{228} \mathrm{Ac} /{ }^{214} \mathrm{Bi}\right), 1173 \mathrm{keV}\left({ }^{60} \mathrm{Co}\right), 1332 \mathrm{keV}\left({ }^{60} \mathrm{Co}\right), 2614 \mathrm{keV}$ $\left({ }^{208} \mathrm{Tl}\right), 4439 \mathrm{keV}\left({ }^{12} \mathrm{C}^{*}\right), 6467 \mathrm{keV}\left({ }^{132} \mathrm{Xe}^{*}\right)$ and $9255 \mathrm{keV}$ $\left({ }^{130} \mathrm{Xe}^{*}\right)$, to their expected values.

The event distributions in $\log _{10}\left(\sum S 2_{b} / \sum S 1\right)$ vs. $E_{\text {corr }}$ for the Run 9 and Run $10 \mathrm{AmBe}$ data are shown in Figure 3. HEGs are selected within a $3 \sigma$ cut on the high energy ER band, and with $E_{\text {corr }}>6.2 \mathrm{MeV}$, about $3 \sigma$ away from $5 \mathrm{MeV}$, as indicated in Figure 3. Some events are observed with lower vertical values than the main ER band. They are all $\alpha$-related events, resulting in smaller ionization signals ( $S 2 \mathrm{~s})$. We categorize the events into three classes according to the vertical values from high to low, i.e., the mixed $\alpha$-ER-events from the internal radon background, the bulk $\alpha \mathrm{s}$, and the wall $\alpha \mathrm{s}$ whose ionization signals cannot be efficiently extracted. The $\alpha$-ER-mixed events have a rate much lower than the AmBeinduced HEG rate here and therefore can be safely omitted.

The number of selected HEG and SSNR events and their ratios are summarized in Table 1, as compared with the simulation to be described in sect. 3.3.

\subsection{AmBe simulation}

A custom Geant4-based program (BambooMC), with the full PandaX-II geometry implemented, is used to simulate energy depositions in the detector by the radiation from the AmBe source. An event generator that samples the neutron energy from Figure 1, with correlated emission of $4.4 \mathrm{MeV} \gamma$ ray for the $\left(\alpha, \mathrm{n}_{1}\right)$ channel, is used to create primary particles.
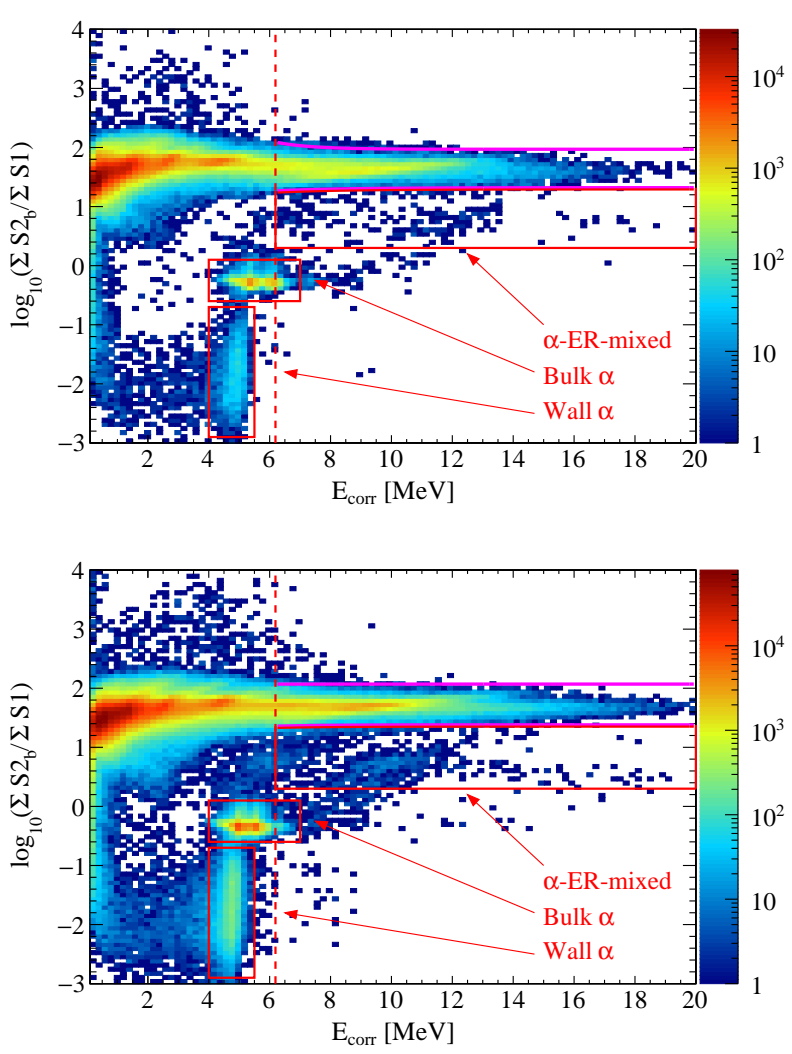

Figure 3 The distributions of events in $\log _{10}\left(\sum S 2_{b} / \sum S 1\right)$ vs. $E_{\text {corr }}$ in the Run 9 (top) and Run 10 (bottom) AmBe data. The $\pm 3 \sigma$ cuts of the ER band are shown as the solid magenta lines. The dashed red lines represent the $6.2 \mathrm{MeV}$ cut for the HEGs. Also indicated below the ER band are the $\alpha$-ER-mixed, bulk $\alpha$ s, and wall $\alpha$ events near the PTFE reflectors.

Table 1 The number of SSNR, HEG events and their ratios in Run 9 and Run $10 \mathrm{AmBe}$ calibration data. The corresponding ratios from the simulation are also listed.

\begin{tabular}{c|ccc|c}
\hline \multirow{2}{*}{ AmBe Run } & \multicolumn{3}{|c|}{ Data } & MC \\
\cline { 2 - 5 } & \# SSNR & \# HEG & Ratio & Ratio \\
\hline Run 9 & 3415 & 49159 & $1 / 14.4$ & $1 / 14.7$ \\
Run 10 & 10390 & 151783 & $1 / 14.6$ & $1 / 15.2$ \\
\hline
\end{tabular}

Note that the correlated $\gamma$ emission in this generator is implemented by hand, effectively equivalent to the improved ( $\alpha$, n) generator discussed later in sect. 4.2. For SSNR events, the energy depositions are converted to simulated $S 1$ and $S 2$ signals using a NEST-based model [19]. For HEG events, instead of running the NEST-based simulation to produce $S 1$ s and $S 2$ s, we simply sum the true energy depositions within the EFV, and make a gentle energy-dependent Gaussian smearing with a $\sigma$ varying from $4 \%$ to $10 \%$ from $1 \mathrm{MeV}$ to $10 \mathrm{MeV}$, to best match the measured HEG spectrum from

2) Since neutron capture in xenon happens within a few $\mu$ s (Figure 5), it is reasonable to simply use $S 1_{\text {max }}$ as the $T_{0}$ of the drift.

3) Note that in the data, the contributions to $S 1$ from the energy depositions inside or outside the EFV cannot be separated. This leads to a slight discrepancy in the energy reconstruction in the data and MC, however its impact is constrained by the spectra comparison in Figure 4. 
the AmBe data. The efficiencies of event detection and selection cuts from $\mathrm{AmBe}$ data are also incorporated into the $\mathrm{MC}$ simulation for the SSNR and HEG events.

The MC simulation is validated in several ways. In Figure 4, the spectra of the measured (non-AmBe background subtracted) and simulated $E_{\text {corr }}$ are overlaid, where the simulation is normalized to the data between 2 and $20 \mathrm{MeV}$. The spectra agree with each other well. A residual difference between Runs 9 and 10 is observed, which is likely from the difference in the PMT saturation because of the varying supply voltages [11]. The fractional difference (data-MC) of the HEGs between 6.2 to $20 \mathrm{MeV}$ is $16.3 \%$ for Run 9 and $18.3 \%$ for Run 10, indicating the level of systematic uncertainties.

To validate the neutron capture process in the $\mathrm{MC}$, we compare the time separation between the prompt and delayed energy deposition in the data and MC. Many neutron events in the data do not have a prompt $S 1$ because the prompt energy is deposited in the dead region. To cleanly select events with a genuine prompt $S 1$, we make a cut at $40 \mathrm{keV}$ in both the data and MC. This energy corresponds to the deexcitation $\gamma$ of ${ }^{129} \mathrm{Xe}$ excited by the neutron. Then we look for the second $S 1$ corresponding to the delayed capture of the same neutron and obtain the distribution of the time difference. A comparison between the data and MC is shown in Figure 5, where one observes a fairly good agreement above $1 \mu \mathrm{s}$. The discrepancy below $1 \mu \mathrm{s}$ is likely due to the inefficiency in identifying two $S$ 1s from the data when they are close-by. On the other hand, the selection of HEGs from the data does not require prompt-delayed S1 pairs, therefore is entirely unaffected.

Lastly, to quantify the global agreement between the $\mathrm{AmBe}$ data and MC, we compare the ratios between SSNR and HEG events in Table 1. For both run periods, the data and MC ratios are consistent within 4\%. An increased number of SSNR events by a factor of $20 \%$ is obtained by an MC simulation without the $4.4 \mathrm{MeV} \gamma$ in the source generator. The difference would further increase when the isolated neutrons are produced at the PTFE or PMTs closer to the xenon target. This demonstrates the importance of correlated $\gamma$ emission in the neutron production model.

\section{The improved simulation}

In this section, we developed an improved MC to calculate the ratio between SSNR and HEG events induced by radiogenic neutrons ( $R_{\mathrm{mc}}$ in Eqn. 2$)$ in the DM data.

\subsection{Evaluation of neutron production rate}

${ }^{238} \mathrm{U},{ }^{235} \mathrm{U}$, and ${ }^{232} \mathrm{Th}$ decay chains are long-lived $\alpha$ emitters. Due to the low Columb potential, $(\alpha, n)$ events are mostly

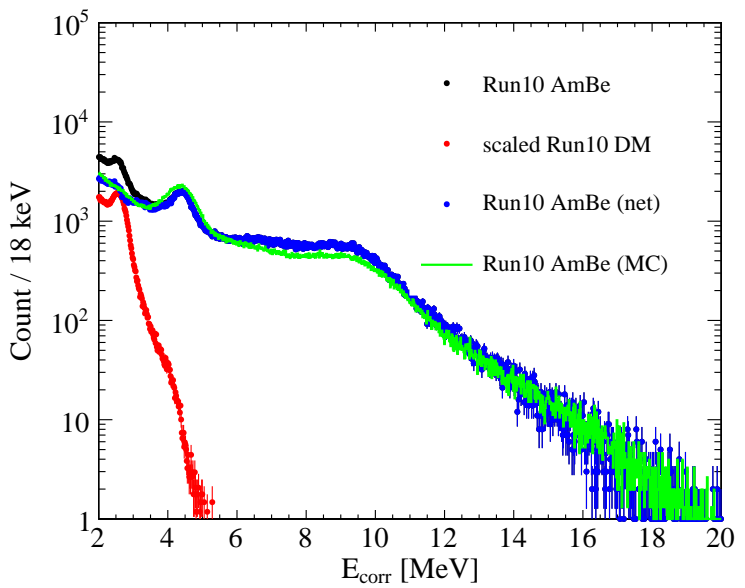

Figure 4 The raw AmBe (black), background (red), and net $\mathrm{AmBe}$ (blue) energy spectra in Run 10. The background spectrum is obtained from the DM data, scaled by the run durations. The net AmBe spectrum is obtained by subtracting the background from the raw AmBe spectrum. The simulated energy spectrum is overlaid in green.

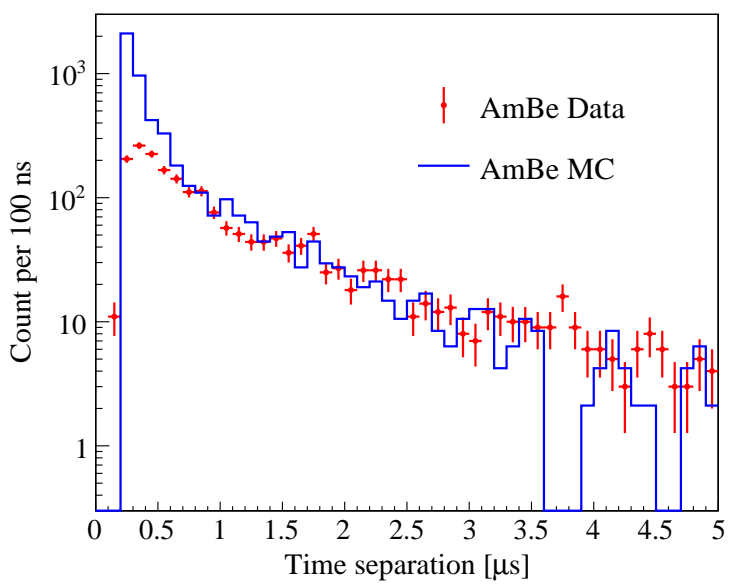

Figure 5 The time separation between the $40 \mathrm{keV}$ prompt $\gamma$ rays from ${ }^{129} \mathrm{Xe}^{*}$ and the delayed HEGs from neutron capture in Run $10 \mathrm{AmBe}$ data (red) and MC (blue).

produced on the light nuclei in the detector, such as fluorine in the PTFE reflector wall, aluminum in the ceramic stem of the PMTs, silicon in the quartz window of the PMTs, etc. In addition, those decay chains also produce SF neutrons. As the input to the simulation, the radioactivities of the most important components for neutron production in the detector are summarized in Table 2.

The neutron yields, $Y_{i j}$, are calculated with SOURCES$4 \mathrm{~A}$ and summarized in Table 3. The SF neutron yield of ${ }^{238} \mathrm{U}_{\mathrm{e}}$ is $1.1 \times 10^{-6}$ neutrons/decay, and that of other chains are negligible. The neutron production rate by each component, $\sum_{j} n_{i j}$, by combining the radioactivities and neutron yields, is also listed in the table, where dominating contribu- 
Table 2 The activities of the materials used for neutron background estimation in the PandaX-II experiment. For ${ }^{238} \mathrm{U}$, its early chain $\left({ }^{238} \mathrm{U}_{\mathrm{e}}\right)$ represents ${ }^{238} \mathrm{U}$ $\rightarrow{ }^{230} \mathrm{Th}$, and the later chain $\left({ }^{238} \mathrm{U}_{\mathrm{l}}\right)$ refers to ${ }^{226} \mathrm{Ra} \rightarrow{ }^{206} \mathrm{~Pb}$. For ${ }^{232} \mathrm{Th}$, the early chain $\left({ }^{232} \mathrm{Th}_{\mathrm{e}}\right)$ and later chain $\left({ }^{232} \mathrm{Th}\right){ }_{\mathrm{l}}$ refer to ${ }^{232} \mathrm{Th} \rightarrow{ }^{228} \mathrm{Ac}$ and ${ }^{228} \mathrm{Th} \rightarrow$ ${ }^{208} \mathrm{~Pb}$, respectively. The activities of the SS and PTFE were measured with the PandaX counting station [20] assuming secular equilibrium. The radioactivities from the 3-in PMTs are taken from Ref. [21].

\begin{tabular}{cc|ccccc}
\hline \multirow{2}{*}{ Component (Material) } & \multirow{2}{*}{ Quantity } & \multicolumn{5}{|c}{ Radioactivity $(\mathrm{mBq} / \mathrm{kg}$ or mBq/piece) } \\
\cline { 3 - 7 } & & ${ }^{238} \mathrm{U}_{\mathrm{e}}$ & ${ }^{238} \mathrm{U}_{\mathrm{l}}$ & ${ }^{235} \mathrm{U}$ & ${ }^{232} \mathrm{Th}_{\mathrm{e}}$ & ${ }^{232} \mathrm{Th}_{1}$ \\
\hline PTFE & $55.5 \mathrm{~kg}$ & 3.2 & 3.2 & 1.3 & 1.5 & 1.5 \\
Cryostat $(\mathrm{SS})$ & $256.9 \mathrm{~kg}$ & 1.7 & 1.7 & 2.4 & 2.7 & 2.7 \\
PMT stem $\left(\mathrm{Al}_{2} \mathrm{O}_{3}\right)$ & 110 piece & 2.4 & 0.3 & 0.1 & 0.2 & 0.1 \\
PMT window $\left(\mathrm{SiO}_{2}\right)$ & 110 piece & 1.2 & 0.07 & 0.02 & 0.03 & 0.03 \\
\hline
\end{tabular}

Table 3 Neutron yields of ${ }^{238} \mathrm{U},{ }^{235} \mathrm{U}$, and ${ }^{232} \mathrm{Th}$ in different materials. Among all channels, fluorine has the largest $(\alpha, \mathrm{n})$ neutron yield. SF only happens in the earl chain, with a yield of about 2 orders less compared to the $(\alpha, \mathrm{n})$. The neutron production rates, $\sum_{j} n_{i j}$, are also listed for corresponding component $i$.

\begin{tabular}{c|ccccc|c}
\hline \multirow{2}{*}{ Material } & \multicolumn{5}{|c|}{ Neutron yield (neutrons/decay) } & \multirow{2}{*}{$\sum_{j} n_{i j}$} \\
& ${ }^{238} \mathrm{U}_{\mathrm{e}}$ & ${ }^{238} \mathrm{U}_{\mathrm{l}}$ & ${ }^{235} \mathrm{U}$ & ${ }^{232} \mathrm{Th}_{\mathrm{e}}$ & ${ }^{232} \mathrm{Th}_{\mathrm{l}}$ & $($ neutrons/day) \\
\hline $\mathrm{PTFE}$ & $7.6 \times 10^{-6}$ & $5.5 \times 10^{-5}$ & $8.9 \times 10^{-5}$ & $7.9 \times 10^{-7}$ & $8.6 \times 10^{-5}$ & $2.1 \times 10^{0}$ \\
$\mathrm{SS}$ & $1.1 \times 10^{-6}$ & $4.1 \times 10^{-7}$ & $3.4 \times 10^{-7}$ & $4.9 \times 10^{-11}$ & $1.5 \times 10^{-6}$ & $1.7 \times 10^{-1}$ \\
$\mathrm{Al}_{2} \mathrm{O}_{3}$ & $1.3 \times 10^{-6}$ & $6.3 \times 10^{-6}$ & $9.5 \times 10^{-6}$ & $1.3 \times 10^{-8}$ & $1.1 \times 10^{-5}$ & $6.6 \times 10^{-2}$ \\
$\mathrm{SiO}_{2}$ & $1.2 \times 10^{-6}$ & $9.9 \times 10^{-7}$ & $1.5 \times 10^{-6}$ & $7.8 \times 10^{-9}$ & $1.6 \times 10^{-6}$ & $1.5 \times 10^{-2}$ \\
\hline
\end{tabular}

tion from PTFE can be observed. The typical contribution to neutron production from SF is about $4 \%$ of that from $(\alpha, \mathrm{n})$.

\subsection{New $(\alpha, \mathbf{n})$ generators}

In recent years, improved low energy nuclear models and data libraries have been incorporated into Geant4 [22]. Therefore, as a remedy to SOURCES-4A, we utilized Geant4 (version $10.03 \mathrm{p} 03)$ and developed a new $(\alpha, \mathrm{n})$ neutron generator to include the excitation/de-excitation of the outgoing nucleus.

In Geant4, the INCL++ model handles the nuclear reactions and G4ExcitationHandler models the nuclear excitations/de-excitations [22]. Long-lived decay chains are launched automatically by Geant4, and for events where the neutron is produced via $(\alpha, \mathrm{n})$, the information of the energy and particle IDs of the production vertices are stored. It is identified that in the native G4ExcitationHandler, the first $\gamma$ in a cascade disrespects the known energy level in the final nucleus. This causes a significant and incorrect suppression for the $\left(\alpha, \mathrm{n}_{0}\right)$ channel relative to the others. A fix is implemented to correct the inappropriate $\gamma$ energy and absorb the difference to the kinetic energy of the neutron ${ }^{4)}$. The corrected kinetic energy of the neutron and different $\gamma$ energies (if any) are then stored and later used as the primary generator of BambooMC. For simplicity, the directions of neutron and $\gamma(\mathrm{s})$ are assumed to be isotropic.

We carried out a BambooMC simulation with the improved generator for ${ }^{238} \mathrm{U}$ in PTFE. The results are summarized in Table 4, together with that by the SOURCES4A generator. Clearly, the former leads to a suppression

\footnotetext{
4) Q. H. Wang, et al., in preparation (2019).
}

Table 4 The $P_{\text {ssnr }}, P_{\text {heg }}$, and their ratio predicted for ${ }^{238} \mathrm{U}$ in PTFE by the two $(\alpha, \mathrm{n})$ generators. $10^{6}$ events are generated in both simulations. See text for details.

\begin{tabular}{cccc}
\hline Generator & $P_{\text {ssnr }}$ & $P_{\text {heg }}$ & Ratio \\
\hline Improved & $1038 / 10^{6}$ & $27602 / 10^{6}$ & $1 / 26.6$ \\
SOURCES-4A & $3997 / 10^{6}$ & $26171 / 10^{6}$ & $1 / 6.5$ \\
\hline
\end{tabular}

in the SSNR events by a factor of four due to the correlated $\gamma$ emission, consistent with the expectation in sect. 3.3.

\subsection{New SF generator}

Similar to the $(\alpha, \mathrm{n})$ reaction, multiple neutrons and $\gamma \mathrm{s}$ are produced in the SF of ${ }^{238} \mathrm{U}$. The average neutron and $\gamma$ multiplicities are 2.0 and 6.4, respectively [23], which was not taken into account in the SOURCES-4A generator. Following the practice in Ref. [23], we employed a special Geant4based program to simulate the SF of ${ }^{238} \mathrm{U}$. This program incorporates the LLNL Fission Library 2.0.2 including a special FREYA model [24] that takes into account the correlations in neutron multiplicity, energy, angles, and the energy sharing between neutrons and $\gamma \mathrm{s}$. Using this new generator with BambooMC, we generated SF of ${ }^{238} \mathrm{U}$ in the PTFE materials, and the resulting SSNR and HEG events are shown in Table 5. Also shown in the same table are those using the SOURCES-4A generator. Due to the large neutron and $\gamma$ multiplicity, the SSNR events are suppressed to a negligible level in comparison to the $(\alpha, \mathrm{n})$ reaction. 
Table 5 The $P_{\text {ssnr }}, P_{\text {heg }}$, and their ratio predicted for ${ }^{238} \mathrm{U}$ in PTFE by the two SF generators. $10^{6}$ events are generated in both simulations. " 2.0 " is the mean number of neutrons per fission. See text for details.

\begin{tabular}{cccc}
\hline Generator & $P_{\text {ssnr }}$ & $P_{\text {heg }}$ & Ratio \\
\hline Improved & $89 / 2.0 / 10^{6}$ & $86693 / 2.0 / 10^{6}$ & $1 / 974.1$ \\
SOURCES-4A & $3885 / 10^{6}$ & $27771 / 10^{6}$ & $1 / 7.1$ \\
\hline
\end{tabular}

\subsection{Evaluation of $R_{\mathrm{mc}}$}

We performed BambooMC simulation for all detector materials in Table 2 with the improved neutron generators. The same cuts used in the analysis of AmBe MC were used to select the SSNR and HEG events. The results are shown in Table 6. Due to the high neutron yield and the high ${ }^{238} \mathrm{U}$ impurity level, the contribution from PTFE dominates the SSNR and HEG events. The naively estimated SSNR background according to Eqn. 1 is about 0.2 events for both Run 9 and Run 10, respectively. As we have mentioned in sect. 2, these values heavily rely on the input of the radioactivities, therefore lack robustness. The overall ratios between the SSNR and HEG events are 1/26.6 for Run 9 and 1/24.6 for Run 10. The difference mainly arises from different FV for SSNR selections in Run 9 and Run 10 [11] (due to different levels of ${ }^{127} \mathrm{Xe}$ background). The uncertainties of $R_{\mathrm{mc}}$ are estimated to be $16.3 \%$ and $18.3 \%$ for Run 9 and Run 10, respectively, based on the spectra difference of HEG events in AmBe data and MC, discussed in sect. 3.3.

\section{Improved neutron background estimation in PandaX-II}

As discussed in Secs. 3.2 and 3.3, neutrons can induce HEG events in xenon target, with a rate much higher than the SSNR events. Therefore in PandaX-II DM data, we look for HEGs and estimate the corresponding SSNR background from detector materials using the $R_{\mathrm{mc}}$ in sect. 4.4.

We selected HEGs from the DM data using the same procedures as those in analyzing the AmBe data. At the high energy region of a few MeVs, the distributions of $\log _{10}\left(\sum S 2_{b} / \sum S 1\right)$ vs. $E_{\text {corr }}$ in Run 9 and Run 10 DM data are shown in Figure 6. The fact that the PMTs in Run 9 and Run 10 were operated at different high voltage [11] leads to different saturation for large $S 1$ s and $S 2$ s, thereby some difference in the high energy event distributions. 36 and 20 HEG candidates are found in Run 9 and Run 10, respectively. However, different from the AmBe data, due to the much lower rate of HEGs, the $\alpha$-ER-mixed events become more pronounced and can contaminate the true HEGs.

Since an $\alpha$ cannot scatter twice in the detector, one expects that the $\alpha$-mixed events contain less amount of $S 2$ s than the
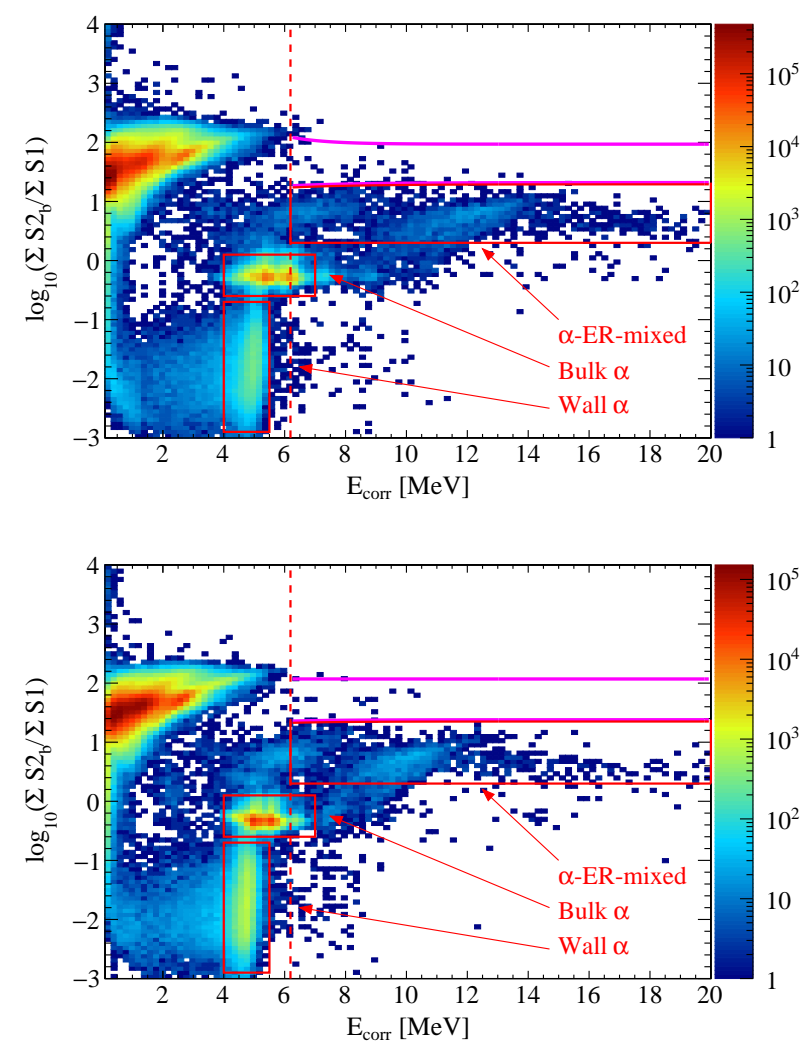

Figure 6 The distributions of events in $\log _{10}\left(\sum S 2_{b} / \sum S 1\right)$ vs. $E_{\text {corr }}$ in the Run 9 (top) and Run 10 (bottom) DM data.

$\gamma$ cascade in the HEGs. This is confirmed further in Figure 7, where the distributions of the number of $S 2 \mathrm{~s}$ vs. $E_{\text {corr }}$ for HEGs in AmBe data and the $\alpha$-mixed events below the ER band in DM data (as indicated in Figure 6) are compared. An $\alpha$-rejection cut is developed (green curve in Figure 7), with an 82\% (61\%) efficiency on HEGs for Run 9 (Run 10) estimated from $\mathrm{AmBe}$ data, with an estimated systematic uncertainty of $10.5 \%$. The corresponding rejection power for $\alpha$-mixed events are $91 \pm 1 \%$ and $89 \pm 1 \%$, respectively, estimated from DM data.

After applying the $\alpha$-rejection cut, the band distributions of the surviving events in Run 9 and Run 10 DM data are shown in Figure 8. In total, 16 and 8 HEG candidates survive in Run 9 and Run 10, respectively. The final HEG candidates have multiple $S 2 \mathrm{~s}$, consistent with those from the AmBe data, with an example waveform shown in Figure 9. The residual $\alpha$-mixed background is estimated to be $1.7 \pm 0.7$ and $0.9 \pm 0.7$ events based on the cut efficiency and rejection power mentioned above. The background subtracted and efficiency corrected HEGs, $N_{\text {heg }}$, are 17.5 \pm 5.6 events in Run 9 and 11.6 \pm 5.7 events in Run 10, where the statistical and systematic uncertainties are combined.

Using $N_{\text {heg }}$ obtained from the data and Eqn. 2, the improved estimation of SSNR background is now $0.66 \pm 0.24$ 
Table 6 Predicted SSNR and HEG events in counts/day and their ratios in PandaX-II using the new generators.

\begin{tabular}{c|ccc|ccc}
\hline \multirow{2}{*}{ Components } & \multicolumn{3}{|c|}{ Run 9 } & \multicolumn{3}{c}{ Run 10 } \\
\cline { 2 - 7 } & \# SSNR & \# HEG & Ratio & \# SSNR & \# HEG & Ratio \\
\hline PTFE & $1.9 \times 10^{-3}$ & $5.8 \times 10^{-2}$ & $1 / 30.8$ & $2.0 \times 10^{-3}$ & $5.8 \times 10^{-2}$ & $1 / 28.8$ \\
Cryostat & $3.4 \times 10^{-4}$ & $2.8 \times 10^{-3}$ & $1 / 8.2$ & $3.5 \times 10^{-4}$ & $2.8 \times 10^{-3}$ & $1 / 7.9$ \\
PMT Stem & $1.2 \times 10^{-4}$ & $1.3 \times 10^{-3}$ & $1 / 10.4$ & $1.6 \times 10^{-4}$ & $1.3 \times 10^{-3}$ & $1 / 7.8$ \\
PMT Window & $5.1 \times 10^{-6}$ & $3.9 \times 10^{-4}$ & $1 / 76.3$ & $6.5 \times 10^{-6}$ & $3.9 \times 10^{-4}$ & $1 / 59.7$ \\
\hline Total & $2.3 \times 10^{-3}$ & $6.2 \times 10^{-2}$ & $1 / 26.6$ & $2.6 \times 10^{-3}$ & $6.3 \times 10^{-2}$ & $1 / 24.6$ \\
\hline
\end{tabular}

and $0.47 \pm 0.25$ events for Run 9 and Run 10, respectively, in which the uncertainties of $N_{\text {heg }}$ and $R_{\mathrm{mc}}$ are combined. The final results are summarized in Table 7 , together with the estimated neutron background from our previous publication [11]. This new evaluation presents a major improvement in the SSNR probability calculation and an in situ normalization against the measured HEGs in the data, therefore supersedes the previous.
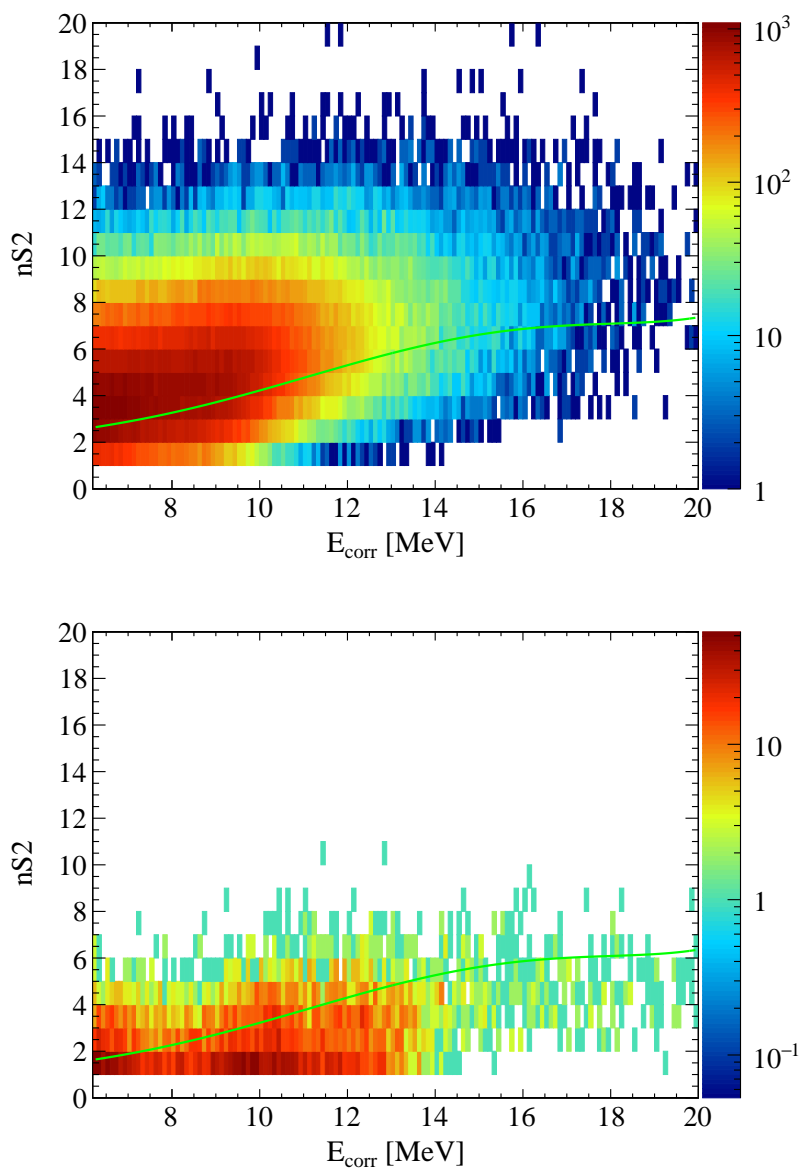

Figure 7 The distribution of number of $S$ 2s vs. $E_{\text {corr }}$ for HEGs in AmBe data (top) and high energy $\alpha$-mixed events in DM data (bottom), Run 9 and Run 10 combined. The green curve represents the $\alpha$-rejection cut.
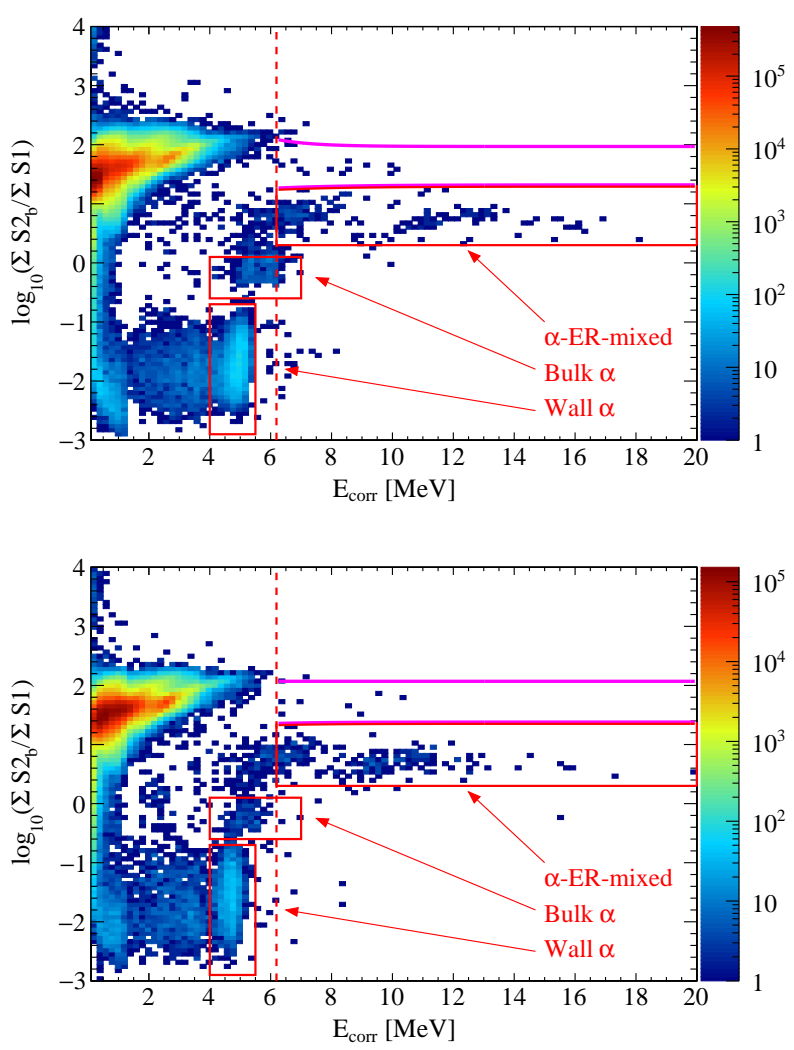

Figure 8 The distribution of $\log _{10}\left(\sum S 2_{b} / \sum S 1\right)$ vs. $E_{\text {corr }}$ for events with the $\alpha$-rejection cut in Run 9 (top) and Run 10 (bottom) DM data.

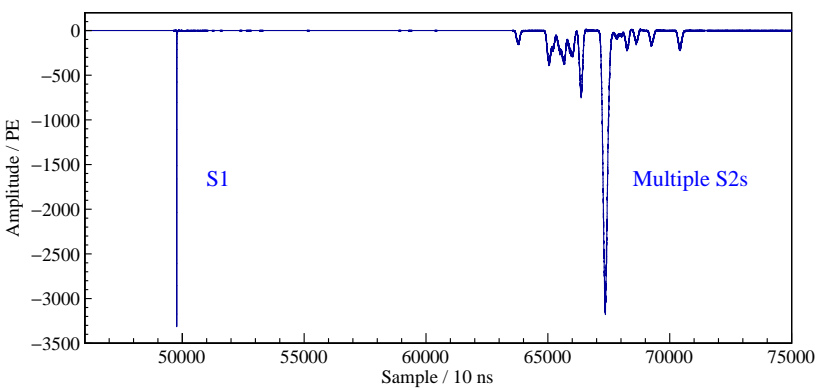

Figure 9 An example HEG waveform from Run 10 DM data. 
Table 7 The neutron background events in Run 9 and Run 10 evaluated with the improved method. The results from our previous publications are shown for comparison.

\begin{tabular}{ccc}
\hline \hline Methods & Run 9 DM & Run 10 DM \\
\hline This work & $0.66 \pm 0.24$ & $0.47 \pm 0.25$ \\
Ref. [11] & $0.85 \pm 0.43$ & $0.83 \pm 0.42$ \\
\hline
\end{tabular}

\section{Summary}

We performed a detailed study on the neutron background originating from detector materials in the PandaX-II experiment. An improved neutron generator model that incorporates the correlated emission of neutron(s) and $\gamma(\mathrm{s})$ was developed and benchmarked. A novel data-driven method, with a robust connection between the SSNR and HEG events, was used to predict the neutron background in PandaX-II. The improved neutron background levels are lower than those reported before and are more reliable with well-controlled uncertainties. In the next generation of PandaX-II, i.e., the 4-ton scale PandaX-4T experiment [25], a solid estimation of the neutron background is expected to be achieved by applying this new method. It can be more generally employed in the neutron background evaluation in other dark matter experiments.

This project has been supported by a Double Top-class grant from Shanghai Jiao Tong University, grants from National Science Foundation of China (Nos. 11435008, 11505112, 11525522, 11775141 and 11755001), a grant from the Ministry of Science and Technology of China (No. 2016YFA0400301). We thank the Office of Science and Technology, Shanghai Municipal Government (No. 11DZ2260700, No. 16DZ2260200, No. 18JC1410200) and the Key Laboratory for Particle Physics, Astrophysics and Cosmology, Ministry of Education, for important support. This work is supported in part by the Chinese Academy of Sciences Center for Excellence in Particle Physics (CCEPP) and Hongwen Foundation in Hong Kong. Finally, we thank the CJPL administration and the Yalong River Hydropower Development Company Ltd. for indispensable logistical support and other help.

1 V. Trimble, Ann. Rev. Astron. Astrophys. 25, 425-472 (1987).

2 M. W. Goodman and E. Witten, Phys. Rev. D 31, 3059-3063 (1985).
3 P. Cushman, et al., Working Group Report: WIMP Dark Matter Direct Detection, in Proceedings, 2013 Community Summer Study on the Future of U.S. Particle Physics: Snowmass on the Mississippi (CSS2013): Minneapolis, MN, USA, July 29-August 6, 2013, arXiv: 1310.8327.

4 J. L. Liu, X. Chen, X. D. Ji, Nature Phys. 13, 212-216 (2017).

5 E. Aprile and T. Doke, Rev. Mod. Phys. 82, 2053-2097 (2010).

6 Y. C. Wu, et al., Chin. Phys. C 37, 086001 (2013).

7 X. G. Cao, et al., Sci. China Phys. Mech. Astron. 57, 1476-1494 (2014).

8 M. J. Xiao, et al. (PandaX Collaboration), Sci. China Phys. Mech. Astron. 57, 2024-2030 (2014).

9 A. D. Tan, et al. (PandaX-II Collaboration), Phys. Rev. Lett. 117, 121303 (2016).

10 T. Zhang, C. B. Fu, X. D. Ji, J. L. Liu, X. Liu, X. M. Wang, C. F. Yao, and X. H. Yuan, J. Inst. 11, T09004 (2016).

11 X. Y. Cui, et al. (PandaX-II Collaboration), Phys. Rev. Lett. 119, 181302 (2017).

12 W. B. Wilson, et al., SOURCES 4A: A Code for Calculating $(\alpha, n)$, Spontaneous Fission, and Delayed Neutron Sources ande Spectra, Technical Report, (Los Alamos National Laboratory, 1999).

13 S. Agostinelli, et al. (GEANT4 Collaboration), Nucl. Instrum. Meth. A 506, 250-303 (2003).

14 J. Allison, et al., IEEE Trans. Nucl. Sci. 53, 270 (2006).

15 K. Shibata, O. Iwamoto, T. Nakagawa, N. Iwamoto, A. Ichihara, S. Kunieda, S. Chiba, K. Furutaka, N. Otuka, T. Ohsawa, T. Murata, H. Matsunobu, A. Zukeran, S. Kamada, and J. Katakura, J. Nucl. Sci. Technol. 48, 1-30 (2011).

16 X. H. Guo, et al. (Daya Bay Collaboration), arXiv:hep-ex/0701029.

17 W. Q. Gu, A study of the Anti-neutrino Flux From the Reaction of the Daya Bay Experiment, Dissertation for the Doctoral Degree, (Shanghai Jiao Tong University, Shanghai, 2018), pp. 137.

18 A. D. Tan, et al. (PandaX-II Collaboration), Phys. Rev. D 93, 122009 (2016).

19 B. Lenardo, K. Kazkaz, A. Manalaysay, J. Mock, M. Szydagis, and M. Tripathi, IEEE Trans. Nucl. Sci. 62, 3387-3396 (2015).

20 X. M. Wang, X. Chen, C. B. Fu, X. D. Ji, X. Liu, Y. J. Mao, H. W. Wang, S. G. Wang, P. W. Xie, and T. Zhang, J. Inst. 11, T12002 (2016).

21 E. Aprile, et al. (XENON1T Collaboration), J. Cosmol. Astropart. Phys. 2016, 027 (2016).

22 GEANT4 Collaboration, Physics Reference Manual Version: geant4 10.3, 2016

23 J. Verbeke, C. Hagmann, and D. Wright, Simulation of Neutron and Gamma Ray Emission from Fission and Photofission. LLNL Fission Library 2.0.2., Technical Report, (Lawrence Livermore National Laboratory, 2016).

24 J. M. Verbeke, J. Randrup, and R. Vogt, Fission Reaction Event Yield Algorithm FREYA 2.0.2 User Manual, Technical Report, Lawrence Livermore National Laboratory, 2017.

25 H. G. Zhang, et al., Sci. China Phys. Mech. Astron. 62, 31011 (2019). 